\title{
Transient finite element modeling of functional electrical stimulation
}

\author{
Nenad D. Filipovic ${ }^{1}$, Aleksandar S. Peulic ${ }^{2}$, Nebojsa D. Zdravkovic ${ }^{3}$, Vesna M. Grbovic-Markovic ${ }^{4}$ \\ and Aleksandra J. Jurisic-Skevin ${ }^{4}$ \\ ${ }^{1}$ Faculty of Mechanical Engineering, University of Kragujevac, 34000 Kragujevac, Serbia \\ 2 Technical Faculty of Cacak, University of Kragujevac, 32000 Cacak, Serbia \\ ${ }^{3}$ Faculty of Information Technology, 11000 Belgrade, Serbia \\ ${ }^{4}$ Medical Faculty Kragujevac, University of Kragujevac, 34000 Kragujevac, Serbia
}

\begin{abstract}
Transcutaneous functional electrical stimulation is commonly used for strengthening muscle. However, transient effects during stimulation are not yet well explored. The effect of an amplitude change of the stimulation can be described by static model, but there is no differency for different pulse duration. The aim of this study is to present the finite element (FE) model of a transient electrical stimulation on the forearm. Discrete FE equations were derived by using a standard Galerkin procedure. Different tissue conductive and dielectric properties are fitted using least square method and trial and error analysis from experimental measurement.

This study showed that FE modeling of electrical stimulation can give the spatial-temporal distribution of applied current in the forearm. Three different cases were modeled with the same geometry but with different input of the current pulse, in order to fit the tissue properties by using transient FE analysis. All three cases were compared with experimental measurements of intramuscular voltage on one volunteer.
\end{abstract}

Key words: Functional electrical stimulation — Transient finite element modeling — Tissue permittivity and conductivity - Fitting tissue properties

\section{Introduction}

Neurophysiologically and anatomically based pain management methods which do not use drugs are an effective alternative to conventional care. Intramuscular stimulation therapy utilizing pin penetration and electrical stimulation are very good technique which can help relieve acute, subacute and chronic neuralgic pain (Ga et al. 2007).

Functional electrical stimulation is the most important application in the field of clinical treatment by currents or magnetism. This technique artificially generates neural activity in order to overcome lost functions of paralyzed, incontinent or sensory impaired persons.

Correspondence to: Nenad Filipovic, Faculty of Mechanical Engineering, University of Kragujevac, S. Janjic 6, 34000 Kragujevac, Serbia

E-mail: fica@kg.ac.rs
A variety of therapeutic techniques and experimental treatment approaches are described by the term "functional electrical stimulation". The improvement of technological aspects and control systems in functional electrical stimulation field is very important. There is a need to have better understanding of the factors that influence the force production capabilities of stimulated muscle, the ability of muscle to produce the desired movement and the metabolic demands of contractions (Manola et al 2005; Stecker 2005). It is very difficult to obtain these factors through in vivo experiments, within vast modalities of the electrical stimulation (Popovic 1990; Sadiku 2000).

A deeper understanding of underlying physical meaning can be done by modeling of an electrical field in simulated human tissue. We investigated the FE methodology which enables complex geometries and utility for wide physical field problems.

The purpose of this paper is to open an avenue for a future specific patient intramuscular stimulation therapy which could be achieved by using FE modeling. 


\section{Materials and Methods}

\section{Finite element modeling}

The geometry of the human forearm is modeled by concentric cylinders for the skin $(1.5 \mathrm{~mm})$, fat $(8.5 \mathrm{~mm})$, muscle $(27.5 \mathrm{~mm})$, cortical bone $(6 \mathrm{~mm})$ and bone marrow layers $(6.5 \mathrm{~mm})$ as it is presented in Fig. 1. A small tapering of cylinder is also considered. The corresponding tissue layer is described by the conductivity $\sigma$ and also the relative permittivity $\varepsilon$. A large range of values for $\sigma$ and $\varepsilon$ at each layer have been published in literature.

We implemented a specific fitting methodology in order to find best fitting values for experimental measurements. Five different materials (skin, fat, muscle, bone and marrow) actually represent ten unknown material properties: five specific conductivities $\sigma_{1}-\sigma_{5}$ and five relative permittivities $\varepsilon_{1}-\varepsilon_{5}$. A global finite element (FE) system (see Appendix I) contains unknown potential per nodes, prescribed potential as boundary conditions and ten unknown material properties inside the matrix system. As we have experimental measurements of intramuscular voltage on and under the skin $v s$. time we have chosen ten equations from global FE system where all ten material properties appeared in order to get standard least square system. All of these calculations are running in time for random chosen ten nodes. Trial and error method is applied in order to estimate these material properties for different time steps. The initial material properties used in this study for different layers are shown in Table 1 (Kuhn and
Table 1. Tissue material constants (Kuhn 2005)

\begin{tabular}{llr}
\hline & $\sigma(\mathrm{S} / \mathrm{m})$ & \multicolumn{1}{c}{$\varepsilon$} \\
\hline skin & 0.00025 & 6000 \\
fat & 0.03 & 25000 \\
muscle & 1 & 120000 \\
bone & 0.02 & 3000 \\
marrow & 0.08 & 10000 \\
\hline
\end{tabular}

$\sigma$, specific conductivity; $\varepsilon$, relative permeability.

Keller 2005). To simplify a problem the dielectric permittivity was supposed to be independent of the frequency.

Experimental measurements with electrical field through forearm have dynamic conditions. We therefore used the dynamic model of the electrical propagation through tissues. This is an improvement of the common implementation of electrical stimulation modeling by static models. Static models neglect the transient aspects of the electrical current and potentials with transient distributions in space. It is especially emphasized at higher frequencies where the transient effects could not be neglected.

The mathematical model for a transient state is described by considering the conservation of the electrical charge, assuming material characteristics to be linear:

$$
\nabla J=-\frac{\partial \rho}{\partial t}, \quad \nabla D=\rho
$$

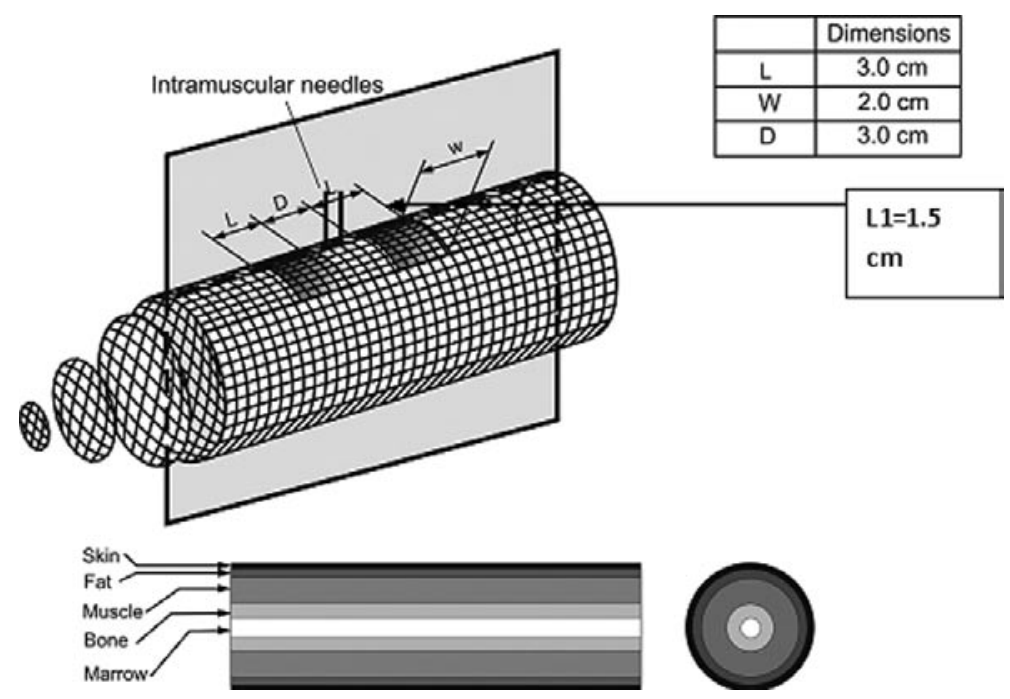

Figure 1. Basic geometrical data for the human forearm. The concentric cylinders denote different tissue layers: skin, fat, muscle, bone and bone marrow. Dimension of stimulated electrodes are $\mathrm{L} \times \mathrm{W}=3 \times 2 \mathrm{~cm}$ and distance between them is $D=3 \mathrm{~cm}$. The position of intramuscular needles is indicated between stimulated electrodes. Also a little tapering of cylinder can be taken into account. Due to plane of symmetry (gray color) for the problem a half forearm is modeled only. (Filipovic et al. 2007 - reproduced from: Journal of the Serbian Society for Computational Mechanics 1, pp. 154-163, with permission). 


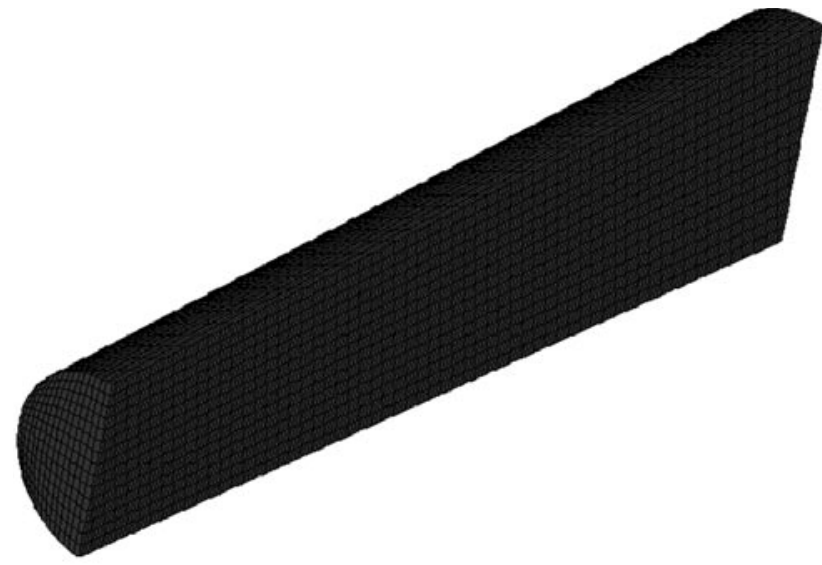

Figure 2. Finite element mesh used for the electro-stimulation modeling, 60000 3D 8-node finite elements. Five different groups of material are associated with the tissue layers.

$$
\begin{aligned}
& D=\varepsilon E, J=\sigma E, E=-\nabla V \\
& \nabla \sigma \nabla V+\nabla \varepsilon \nabla \frac{\partial V}{\partial t}=0
\end{aligned}
$$

where $J$ is the electrical current density vector, $\rho$ represents the electrical charge density (volume), $D$ stands for dielectric displacement vector, $E$ represents electrical field strength vector, $V$ stands for electrical potential, $\varepsilon$ is dielectric permittivity, and $\sigma$ is electrical conductivity (Kuhn and Keller 2005). The Eq. (3) is a direct consequence of Eqs. (1) and (2).

A standard Galerkin procedure is applied to Eq. (3) in order to derive the FE equations (Filipovic et al. 2006; Kojic et al. 2008). The unsteady equations for FE solver were obtained

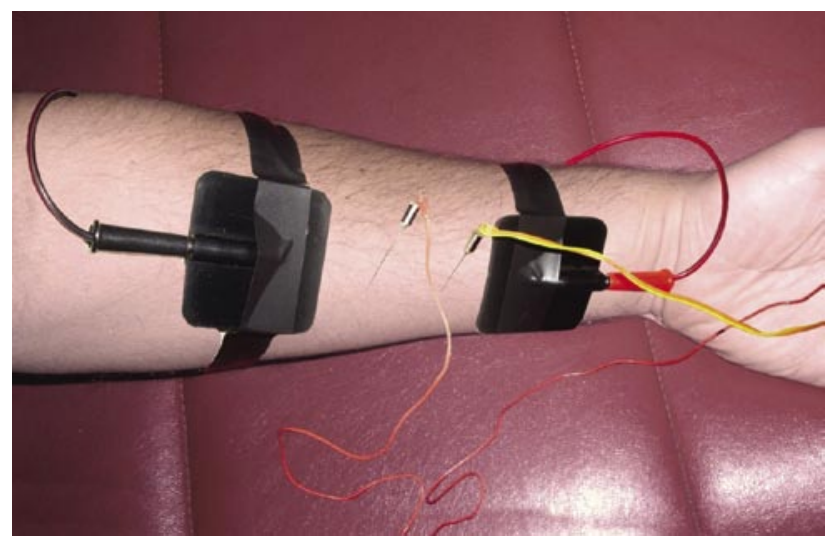

Figure 3. Experimental setup for electrical stimulation. Two electrodes of size $2 \times 3 \mathrm{~cm}$ are connected to a current regulated electric stimulator. The distance between electrodes is $3 \mathrm{~cm}$. The distance between needles is $\mathrm{L} 1=1.5 \mathrm{~cm}$. The voltage between electrodes is measured with the standard equipment (Peulic and Filipovic 2007).

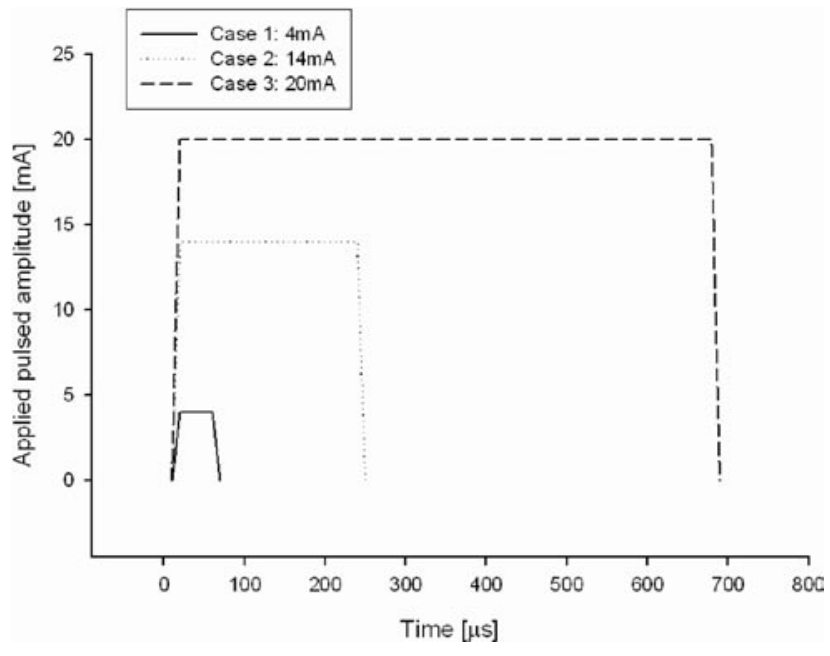

Figure 4. Prescribed current pulses for three cases. The current for the Case $1, \mathrm{I}=4 \mathrm{~mA}$ with periodic pulse duration $50 \mu \mathrm{s}$; for the Case 2 the current $\mathrm{I}=14 \mathrm{~mA}$ with periodic pulse duration $230 \mu \mathrm{s}$; and for Case 3 the current $\mathrm{I}=20 \mathrm{~mA}$ with periodic pulse duration $670 \mu \mathrm{s}$.

by modification of our own FE software package, which is developed at University of Kragujevac (Filipovic et al. 2007).

The FE mesh which consists of 60000 3D 8-node elements is shown in Fig. 2. The unknown values per node are the electrical potentials. To do a mesh independent analysis we examined three different mesh sizes associated with 60000 , 80000 and $100000 \mathrm{FE}$. All of them gave similar results with no significant changes, so we present here the results using 60000 3D FE (Fig. 2).

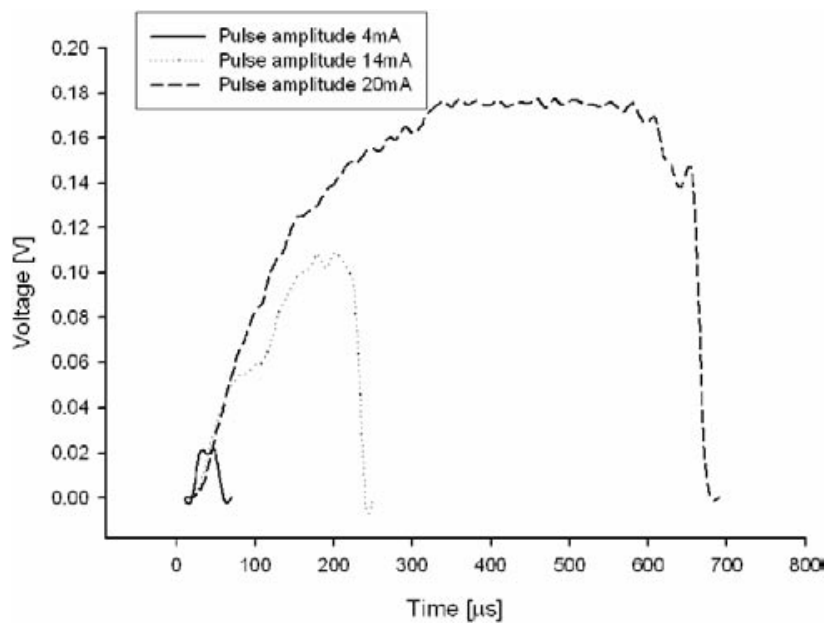

Figure 5. Intramuscular voltage $1 \mathrm{~cm}$ under the skin $v s$. time for three different applied pulse amplitudes: 4, 14 and $20 \mathrm{~mA}$. Pulse duration $t=50,230,670 \mu$ s; two electrodes of size $3 \times 2 \mathrm{~cm}$ with distance between them $3 \mathrm{~cm}$ were used. 

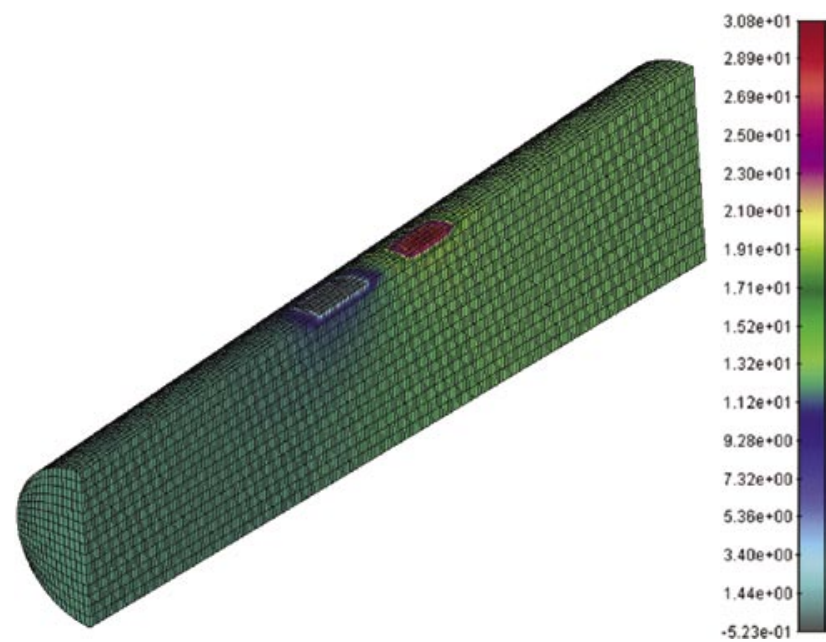

Figure 6. Electrical potential distribution (in volts) at the end of pulse in the Case 3. The pulse duration $t_{p}=670 \mu \mathrm{s}$. Two electrodes of size $3 \times 2 \mathrm{~cm}$ with distance between them $3 \mathrm{~cm}$ are implemented.

We implemented the constant shapes and sizes of the electrodes for stimulation into the FE model as well as same position of intramuscular needles, in order to better fit tissue properties from our own experimental observations.

Boundary conditions for numerical models are determined by pulsed current density at the both electrodes while zero potential is prescribed at one of them as ref-

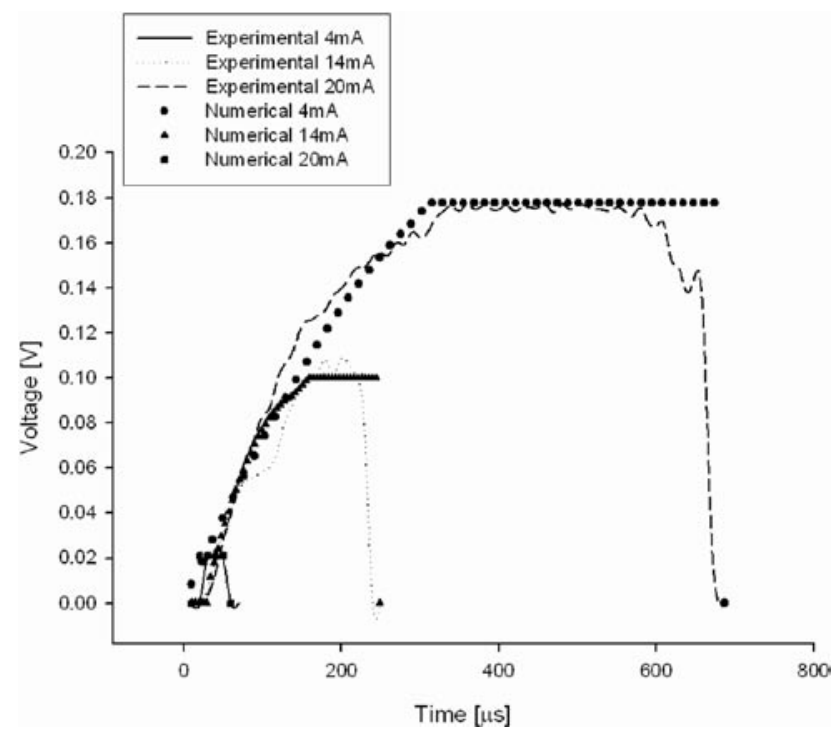

Figure 8. Intramuscular voltage distribution $1 \mathrm{~cm}$ under the skin $v s$. time (all three cases, experimental and computed values). The applied pulse amplitudes: 4, 14 and $20 \mathrm{~mA}$; pulse duration: $\mathrm{t}=$ 50,230 and $670 \mu \mathrm{s}$; two electrodes of size $2 \times 3 \mathrm{~cm}$ with distance between them $3 \mathrm{~cm}$ were used.

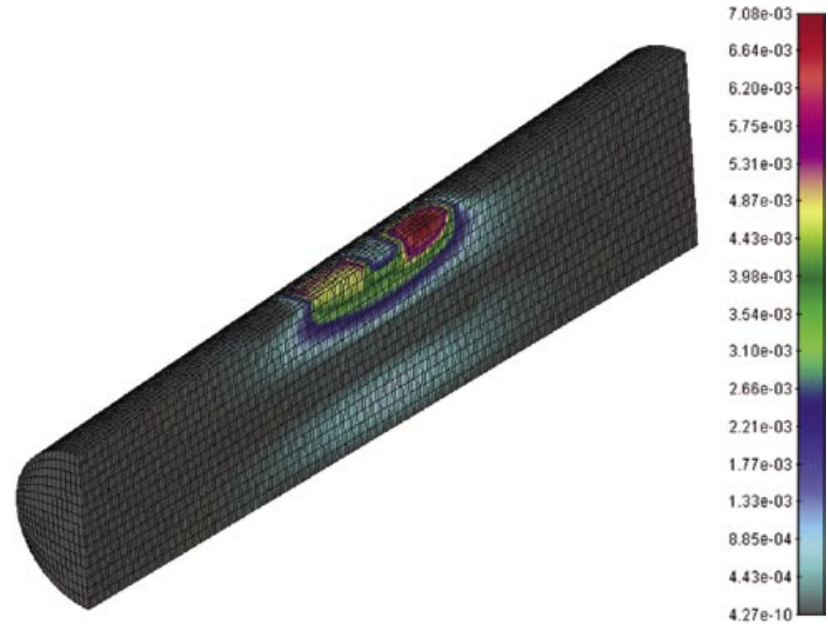

Figure 7. Current density distribution (in $\mathrm{A} / \mathrm{cm}^{2}$ ) inside the human forearm at the end of pulse in the Case 3. The pulse duration $t_{p}=670 \mu s$.

erence point. All other nodes are left with free degree of freedom which means unknown electrical potential values. The current density $J$ as an input flux is directly calculated from the given current amplitude and surface of the electrodes.

\section{Experimental Setup}

An experimental setup was designated to make electrical stimulation on a human volunteer as it is shown in Fig. 3. A standard current regulated stimulator was used (Nedeljkovic et al. 2007; Peulic and Filipovic 2007). The pulse duration during the experiment which we denoted as case 1 was 50 $\mu$ s and the current amplitude was $4 \mathrm{~mA}$ (see Fig. 4). In the next two cases ( 2 and 3 ) the input current pulses were $14 \mathrm{~mA}$ and $20 \mathrm{~mA}$ with $200 \mu$ s and $670 \mu$ s duration. The reason for different duration was a volunteer accommodation to the experiment setup as well as better fitting with numerical simulation. The material of electrode is flexible carbon rubber $25 \times 35 \mathrm{~mm}$. For intramuscular voltage measurement we used single fiber EMG needles of lenght $40 \mathrm{~mm}$. The distance between needles was $\mathrm{L} 1=1.5 \mathrm{~cm}$. The electrolyte between electrode and skin was water. We neglected the voltage drop across impedance of the electrodes.

\section{Results}

We first present the experimental results for intramuscular voltage $1 \mathrm{~cm}$ under the skin $v s$. time for three different applied pulse amplitudes: 4, 14 and $20 \mathrm{~mA}$ (see Fig. 5). Also pulse duration was different for each applied current $t=50,230$ and $670 \mu$ s. 
Table 2. Tissue material constants fitted with our experimental setup

\begin{tabular}{llr}
\hline & $\sigma(\mathrm{S} / \mathrm{m})$ & \multicolumn{1}{c}{$\varepsilon$} \\
\hline skin & 0.00032 & 8200 \\
fat & 0.012 & 22000 \\
muscle & 0.83 & 90000 \\
bone & 0.044 & 5200 \\
marrow & 0.12 & 11000 \\
\hline
\end{tabular}

$\sigma$, specific conductivity; $\varepsilon$, relative permeability.

Electrical potential distribution for maximum voltage between two electrodes on the skin is shown in Fig. 5. As it is expected, the maximum voltage occurred at the second half phase of input current pulse $t_{p}=670 \mu \mathrm{s}$. Also, both calculations demonstrate the strong influence of the transient conditions which is caused by the inertial effects of the voltage response on the skin as well as in the muscle and the nerves. This directly emphasize that permittivity plays an important role in the functional electrical stimulation.

The voltage distribution inside muscle and other tissue layers for the maximum pulse in the case 3 is shown in Fig. 6 . These values are smaller than the voltage on the skin due to low skin conductivity. Current density distribution for the same time step is shown in Fig. 7. As expected, the electrical field produces the current density flow from input to output electrode, caused by electrical potential difference at the electrodes.

Comparison for voltage distribution of numerical results and experimental data for all three cases is shown in Fig. 8. A specific trial-and-error algorithm in combination with least square method on ten nodes from FE mesh is implemented in order to determine corresponding parameters for tissue properties. This comparison shows good agreement. The fitted conductivities and permittivities are presented in Table 2.

\section{Discussion and Conclusion}

To fit tissue material properties of human forearm on volunteer who is stimulated with pulsed current density a transient 3D FE model is developed. We analyzed three cases, with the same geometrical data but different input current amplitude, pulse duration, and fitted tissue properties. The main idea was to fit tissue properties in order to obtain the results close to the experimental response measured on volunteer. All three cases have shown a strong inertial effect due to a transient conditions and dominant relative permittivity of the skin. This observation is especially dominant for short pulsed amplitude where a transient FE modeling becomes very important. The dielectric tissue properties (permittivity) cannot be neglected and the static approach of electrical stimulation should be replaced with the dynamic one.
Kuhn and Keller also analysed transient effect on electrical stimulation in three human volunteers but they did not implement numerical fitting procedure for material properties (Kuhn and Keller 2005). The difference between our previous publication (Filipovic et al. 2007) and our previous study is that we fitted intramuscular voltage now with specific numerical procedure using least square method on ten random chosen FE nodes. The current therapeutic strategy and potential measurement during the functional electrical stimulation may change in the direction of specific patient tissue material properties which can be fitted with FE modeling.

Acknowledgement. This study is partly supported by Ministry of Science in Serbia with projects OI144028 and TR12007.

\section{Appendix I}

The mathematical model for a transient state of electrical stimulation is described by the following equation (Eq. (3) from section Materials and Methods):

$$
\nabla \sigma \nabla V+\nabla \varepsilon \nabla \frac{\partial V}{\partial t}=0
$$

Using Eq. (A.1) a standard Galerkin procedure could be implemented to get discretize finite element system (Kojic et al. 2008). The procedure is the following:

$$
\begin{aligned}
& \int_{V}\left[\frac{\partial}{\partial x}\left(H \sigma \frac{\partial V}{\partial x}\right)+\frac{\partial}{\partial y}\left(H \sigma \frac{\partial V}{\partial y}\right)+\frac{\partial}{\partial z}\left(H \sigma \frac{\partial V}{\partial z}\right)\right] d V+ \\
& +\int_{V}\left[\frac{\partial}{\partial x}\left(H \varepsilon \frac{\partial \dot{V}}{\partial x}\right)+\frac{\partial}{\partial y}\left(H \varepsilon \frac{\partial \dot{V}}{\partial y}\right)+\frac{\partial}{\partial z}\left(H \varepsilon \frac{\partial \dot{V}}{\partial z}\right)\right] d V=0
\end{aligned}
$$

By applying integration by part and Gaussian conversion of volume to surface integral and assuming that $\sigma$ and $\varepsilon$ are constant, Eq. (A.2) could be written in form:

$$
\begin{aligned}
& \int_{V} \sigma\left[\frac{\partial H}{\partial x} \frac{\partial V}{\partial x}+\frac{\partial H}{\partial y} \frac{\partial V}{\partial y}+\frac{\partial H}{\partial z} \frac{\partial V}{\partial z}\right] d V+ \\
& \int_{V} \varepsilon\left[\frac{\partial H}{\partial x} \frac{\partial \dot{V}}{\partial x}+\frac{\partial H}{\partial y} \frac{\partial \dot{V}}{\partial y}+\frac{\partial H}{\partial z} \frac{\partial \dot{V}}{\partial z}\right] d V= \\
& =\int_{S} \sigma H\left(\frac{\partial V}{\partial x} n_{x}+\frac{\partial V}{\partial y} n_{y}+\frac{\partial V}{\partial z} n_{z}\right) d S+ \\
& \int_{S} \varepsilon H\left(\frac{\partial \dot{V}}{\partial x} n_{x}+\frac{\partial \dot{V}}{\partial y} n_{y}+\frac{\partial \dot{V}}{\partial z} n_{z}\right) d S
\end{aligned}
$$

If Eq. (A.3) uses form of potential at the end of the current time step $\mathrm{t}+\Delta \mathrm{t}{ }^{t+\Delta t} V$ the following equation is getting: 


$$
\begin{aligned}
& \int_{V}\left[\frac{\partial H}{\partial x} \frac{\partial H}{\partial x}+\frac{\partial H}{\partial y} \frac{\partial H}{\partial y}+\frac{\partial H}{\partial z} \frac{\partial H}{\partial z}\right] d V\left\{\sigma^{t+\Delta t} V+\varepsilon^{t+\Delta t} \dot{V}\right\} \\
& =\int_{S} F^{S} d S
\end{aligned}
$$

where $F^{S}$ is surface boundary condition for potential field.

If we use time derivative potential at the end of the current time step ${ }^{t+\Delta t} \dot{V}=\left({ }^{t+\Delta t} V-{ }^{t} V\right) / \Delta t$ we will get:

$$
\begin{aligned}
& \int_{V}\left[\frac{\partial H}{\partial x} \frac{\partial H}{\partial x}+\frac{\partial H}{\partial y} \frac{\partial H}{\partial y}+\frac{\partial H}{\partial z} \frac{\partial H}{\partial z}\right] d V \\
& \left\{\sigma^{t+\Delta t} V+\varepsilon\left(\frac{{ }^{t+\Delta t} V-{ }^{t} V}{\Delta t}\right)\right\}=\int_{S} F^{S} d S
\end{aligned}
$$

Derivation in Eq. (A.5) is on the single finite element. After assembling all the finite elements to global system of nodes from 1 to $\mathrm{n}$ we will get:

$$
\left\{\begin{array}{cccc}
K_{11} & K_{12} & \ldots & K_{1 n} \\
K_{21} & \ldots & \ldots & K_{2 n} \\
\ldots & \ldots & \ldots & \ldots \\
K_{n 1} & K_{n 2} & \ldots & K_{n n}
\end{array}\right\}\left\{\begin{array}{c}
V_{1} \\
V_{2} \\
\ldots \\
V_{n}
\end{array}\right\}=\left\{\begin{array}{c}
F_{1} \\
F_{2} \\
\ldots \\
F_{n}
\end{array}\right\}
$$

where $V_{1}, V_{2}, \ldots V_{n}$ are global potential unknowns per finite element nodes.

When incorporated the boundary condition for prescribed electrical potential on the boundary the system of equation (A.6) becomes

$$
\left\{\begin{array}{cccc}
K_{11} & K_{12} & \ldots & K_{1 n} \\
K_{21} & \ldots & \ldots & K_{2 n} \\
\ldots & \ldots & K_{i i}^{*} & \ldots \\
\ldots & \ldots & \ldots & \ldots \\
K_{n 1} & K_{n 2} & \ldots & K_{n n}
\end{array}\right\}\left\{\begin{array}{c}
V_{1} \\
\ldots \\
V_{i}^{*} \\
\ldots \\
V_{n}
\end{array}\right\}=\left\{\begin{array}{c}
F_{1} \\
F_{2} \\
\ldots \\
\ldots \\
F_{n}
\end{array}\right\}
$$

where $V_{i}^{*}$ is prescribed potential as Dirichlet boundary condition at node " $i$ ".

As we have five different materials (skin, fat, muscle, bone and marrow) there are ten unknown material properties: five specific conductivity $\sigma_{1}-\sigma_{5}$ and five relative permittivity $\varepsilon_{1}-\varepsilon_{5}$ for total finite element system. So we can arrange the system of equations from (A.7) to

$$
\left\{\begin{array}{cccc}
\tilde{K}_{11} & \tilde{K}_{12} & \ldots & \tilde{K}_{1 n} \\
\tilde{K}_{21} & \ldots & \ldots & \tilde{K}_{2 n} \\
\ldots & \ldots & \tilde{K}_{i i} & \ldots \\
\ldots & \ldots & \ldots & \ldots \\
\tilde{K}_{101} & \tilde{K}_{102} & \ldots & \tilde{K}_{10 n}
\end{array}\right\}\left\{\begin{array}{c}
V_{1}^{*} \\
V_{2}^{*} \\
\ldots \\
V_{9}^{*} \\
V_{10}^{*}
\end{array}\right\}=\left\{\begin{array}{c}
\tilde{F}_{1} \\
\tilde{F}_{2} \\
\ldots \\
\ldots \\
\tilde{F}_{10}
\end{array}\right\}
$$

where $\tilde{K}_{i i}$ are coefficients of matrix which include the specific conductivity $\sigma_{1}-\sigma_{5}$ and relative permittivity $\varepsilon_{1}-\varepsilon_{5}$. Obviously the vector of prescribed nodal potential in (A.8) is known and the material properties are not known so we can arrange the system in (A.8) into different form:

$$
\left\{\begin{array}{cccc}
\hat{K}_{11} & \hat{K}_{12} & \ldots & \hat{K}_{1 n} \\
\hat{K}_{21} & \ldots & \ldots & \hat{K}_{2 n} \\
\ldots & \ldots & \hat{K}_{i i} & \ldots \\
\ldots & \ldots & \ldots & \ldots \\
\hat{K}_{101} & \hat{K}_{102} & \ldots & \hat{K}_{10 n}
\end{array}\right\}\left\{\begin{array}{c}
\sigma_{1} \\
\sigma_{2} \\
\ldots \\
\varepsilon_{4} \\
\varepsilon_{5}
\end{array}\right\}=\left\{\begin{array}{c}
0 \\
0 \\
\ldots \\
\ldots \\
0
\end{array}\right\}
$$

As we can see from (A.9) the rectangle system of equations could be written in form:

$$
[\hat{K}] \times\left\{\sigma_{\varepsilon}\right\}=\{0\}
$$

where $[\hat{K}]$ matrix is dimension of $10 \times \mathrm{n}$. In order to implement the least square method for Eq. (A.10) we have to multiple both side with transposed matrix of $[\hat{K}]$, so we get:

$$
[\hat{K}]^{T}[\hat{K}] \times\left\{\sigma_{\varepsilon}\right\}=[\hat{K}]^{T}\{0\}
$$

And a square system of equations is getting which actually represents the least square method to find the vector of parameters $\left\{\sigma_{\varepsilon}\right\}^{T}=\left\{\sigma_{1}, \sigma_{2}, \sigma_{3}, \sigma_{4}, \sigma_{5}, \varepsilon_{1}, \varepsilon_{2}, \varepsilon_{3}, \varepsilon_{4}, \varepsilon_{5}\right\}$

As we have transient calculation the system of (A.11) is solved in a some characteristics time steps in the pulse (for example $\mathrm{T} / 4, \mathrm{~T} / 2,3 \mathrm{~T} / 4$ ) by calculated matrix $[\hat{K}]$ using transient definition in Eq. (A.5).

Finally data for getting the vector of material properties are averaged during the time and summarized (see Table 2 in the section Results).

\section{References}

Alo K. M., Holsheimer J. (2002): New trends in neuromodulation for the management of neuropathic pain. Neurosurgery 50, 690-703 doi:10.1097/00006123-200204000-00003

Filipovic N., Mijailovic S., Tsuda A., Kojic M. (2006): An implicit algorithm within the arbitrary Lagrangian-Eulerian formulation for solving incompressible fluid flow with large boundary motions. Comp. Meth. Appl. Mech. Eng. 195, 6347-6361 doi:10.1016/j.cma.2005.12.009

Filipovic N., Nedeljkovic M., Peulic A. (2007): Finite element modeling of a transient functional electrical stimulation. Journal of the Serbian Society for Computational Mechanics 1, 154-163 
Ga H., Koh H. J., Choi J. H., Kim C. H. (2007): Intramuscular and nerve root stimulation vs lidocaine injection to trigger points in myofascial pain syndrome. J. Rehabil. Med. 39, 374-382 doi:10.2340/16501977-0058

Kojic M., Filipovic N., Stojanovic B., Kojic N. (2008): Computer Modeling in Bioengenineering: Theoretical Background, Examples and Software. John Wiley and Sons Press, Chichester, England

Kuhn A., Keller T. A. (2005): A 3D transient Model for transcutaneous functional electrical stimulation. 10th Annual Conference of the International FES Society, Montreal, Canada

Manola L., Roelofsen B. H., Holsheimer J., Marani E., Geelen J. (2005): Modelling motor cortex stimulation for chronic pain control: electrical potential field, activating functions and responses of simple nerve fibre models. Med. Biol. Eng. Comput. 43, 335-343 doi:10.1007/BF02345810

Nedeljkovic M., Filipovic N., Koelndorfer J., Steiner M., Vujanić A. (2007): Finite element modeling of electrical stimulation on lower arm. Biomechanics Symposium, First Serbian Congress on Theoretical and Applied Mechanics, Kopaonik, Serbia

Peulic A., Filipovic N. (2007): Device design for the electrical stimulation. 51st ETRAN conference (Electronics, Telecommunications, Computers, Automatic Control and Nuclear Engineering), Herceg-Novi, Serbia, June 2007

Sadiku M. N. O. (2000): Numerical Techniques in Electromagnetics. CRC Press LCC, N.W. Corporate Blvd., Boca Raton, Florida Stecker M. M. (2005): Transcranial electric stimulation of motor pathways: a theoretical analysis. Comput. Biol. Med. 35, 133-155

doi:10.1016/j.compbiomed.2003.12.005

Received: March 28, 2010

Final version accepted: July 21, 2010 